FTUV-08/0218, IFIC-08-11

Feb. 18, 2008

\title{
BPS Preons and the AdS-M-algebra
}

\author{
Igor A. Bandos ${ }^{\dagger *}$ and José A. de Azcárraga ${ }^{\dagger}$ \\ † Departamento de Física Teórica, Univ. de Valencia and IFIC (CSIC-UVEG), \\ 46100-Burjassot (Valencia), Spain \\ *Institute for Theoretical Physics, NSC Kharkov Institute of Physics and Technology, \\ UA61108, Kharkov, Ukraine
}

\begin{abstract}
We present here the AdS generalization of BPS preons, which were introduced as the hypothetical constituents of M-theory preserving all but one supersymmetries. Our construction, suggested by the relation of 'lower dimensional preons' with higher spin theories, can be considered as a deformation of the M-algebraic description of the single supersymmetry broken by a preon, and provides another reason to identify the AdS generalization of the M-algebra, which we call the AdS-M-algebra, with osp(1|32).
\end{abstract}




\section{Introduction}

Preons were introduced [1] as the possible fundamental constituents of M-theory. They are defined as BPS states that preserve all supersymmetries but one. For $D=11$, this means 31 supersymmetries out of 32 , and hence a preon may be labelled as

$$
\mid B P S \text { preon }>=\mid B P S, 31 / 32>\text {. }
$$

As shown in [1], a $k / 32$-BPS state for $1<k<32$ may be considered as a composite of $\tilde{n}=32-k$ preons. Fully supersymmetric BPS states $(k=32)$ do not contain any preons and, hence, may be considered as preonic vacua ('vacua of vacua', since all the $k$-supersymmetric BPS states are stable and are considered themselves as different M-theory vacua); a preon is the simplest excitation over such a fully supersymmetric vacuum. At the other extreme, a nonsupersymmetric (and, hence, non BPS) state, breaking all 32 supersymmetries, is a composite of the maximal number, 32, of independent BPS-preons.

The preon definition [1] also applies to arbitrary $D$ [2, 3]. The $D=4,6,10$ counterparts of a BPS preon can be associated [2, 3, 4, with an infinite tower of free higher spin fields (see [5, 6]). This identification can be established through the quantization [7, 4] of a generalized superparticle [8] which provides a model for a point-like or 0-brane preon [2, 3, 9].

The standard realization of BPS states is provided by $k$-supersymmetric solutions of the equations of motion for the $D=11$ or type II $D=10$ supergravities, which are low energy limits of M-theory 1 . A $k$-supersymmetric BPS state, or $k / 32$-BPS state, may be described by a supergravity solution preserving a fraction $k / 32$ of the supersymmetries. The $k$-supersymmetric bosonic solutions are characterized by $k$ bosonic Killing spinors, which obey the generalized Killing spinor equation

$$
\mathcal{D} \epsilon_{I}^{\alpha}:=D \epsilon_{I}^{\alpha}+\epsilon_{I}^{\beta} t_{\beta}^{\alpha}:=d \epsilon_{I}^{\alpha}+\frac{1}{4} \epsilon_{I}^{\beta} \Gamma_{a b \beta}{ }^{\alpha} \omega^{a b}-\epsilon_{I}{ }^{\beta} t_{\beta}^{\alpha}=0, \quad I=1, \ldots, k .
$$

In eq. (2), $\mathcal{D}=d-w=D-t$ is the generalized covariant derivative involving the generalized connection $w_{\beta}{ }^{\alpha}=\omega_{\beta}{ }^{\alpha}+t_{\beta}{ }^{\alpha}$, where $\omega_{\beta}{ }^{\alpha}=\frac{1}{4} \omega^{a b} \Gamma_{a b \beta}{ }^{\alpha}$ is the spin connection and $t_{\beta}{ }^{\alpha}$ is the tensorial contribution constructed from the fluxes (the field strengths of the gauge fields in the supergravity multiplets). In $D=11$ supergravity [10] this tensorial contribution reads

$$
t_{\beta}^{\alpha}=\frac{i}{18} e^{a} F_{a b_{1} b_{2} b_{3}} \Gamma^{b_{1} b_{2} b_{3}}{ }_{\beta}^{\alpha}+\frac{i}{144} e^{a} \Gamma_{a b_{1} b_{2} b_{3} b_{4} \beta^{\alpha}} F^{b_{1} b_{2} b_{3} b_{4}},
$$

where $F_{4}=d C_{3}=\frac{1}{4} e^{c_{4}} \wedge \ldots \wedge e^{c_{1}} F_{c_{1} \ldots c_{4}}$ is the field strength of the three-form gauge field $C_{3}$. In $D=11$, eq. (2) is the only restriction for Killing spinors, while in $D=10$ type II and lower dimensional cases, they also have to satisfy an algebraic equation, $\epsilon_{I}{ }^{\alpha} \mathcal{M}_{\alpha \beta}=0$, where the matrix $\mathcal{M}_{\alpha \beta}$ is constructed from the scalars and the field strengths (fluxes) of the gauge fields of the corresponding supergravity multiplets. A hypothetical preonic solution (for $D=11$ or IIA, IIB for $D=10$ ) would have 31 Killing spinors, $\epsilon_{I}{ }^{\alpha}$. Since there is only one bosonic spinor $\lambda_{\alpha}$ orthogonal to all of them,

$$
\epsilon_{I}^{\alpha} \lambda_{\alpha}=0, \quad I=1, \ldots, 31, \quad \alpha=1, \ldots, 32,
$$

a preonic supergravity solution may also be characterized by such a preonic spinor $\lambda_{\alpha}$.

Algebraically (i.e., from the structure of the M-theory superalgebra or 'M-algebra' [11]), any $k / 32$ is allowed for a BPS state [12, 13. However, only (bosonic) solutions for the following number of preserved supersymmetries have been found at present

$$
k=0,1,2,3,4,5,6,8,10,12,14,16,18,20,22,24,26,28, \quad 32
$$

${ }^{1}$ We will not consider here the $\mathrm{N}=1, D=10$ supergravity-SYM interacting systems describing the low energy limits of the two heterotic strings and type I 'corners' of M-theory. 
(see e.g. [14] for further discussion); the preonic solution is conspicuously missing in this list.

The interest on the possible existence of 31/32-supersymmetric or preonic solutions began around 2003 [15, 16, 9]. Recently, a series of no-go results have been obtained for the 'free', 'classical' $D=11$ and $D=10$ type II supergravities [17, 18, 19, 20] 2. However, for supergravity with $\left(\alpha^{\prime}\right)$ corrections the existence of preonic solutions is still open 3 (see [18] and [22] for further discussion). Moreover, even the possible absence of preonic solutions in the presence of corrections or sources from superbranes would not preclude the preon hypothesis, as such a 'preon conspiracy' would still allow us to consider all supersymmetric BPS states as composites of preons (in the same way as, by way of an analogy, quark confinement does not prevent the existence of quarks). Although a dynamical mechanism to construct $k / 32$-BPS states out of 31/32-preons is lacking, a further study of the properties of preons may shed light in this direction. With this in mind, we consider in this paper the problem of AdS generalization of BPS preon. Not surprisingly, the AdS preon will turn out to be related to the description of free massless conformal AdS higher spin theories [23, 24] in the generalized AdS superspaces given by the $O S p$ supergroup manifolds [25, 26, 27, 28].

Let us go back to the idea of preons as elementary excitations over a fully supersymmetric vacuum. The supergravity solutions that describe fully supersymmetric BPS states include [29], besides the Minkowski vacuum of superPoincaré symmetry, the $A d S_{(p+2)} \times S^{(D-p-2)}$ spaces, $(D, p)=(11,2),(11,5),(10,3)$, and the $p p$-wave spaces which will not be considered here. Thus, preons may correspond to the simplest excitations over the Minkowski vacuum or over an $A d S \times S$ vacuum. However, their original definition referred to the M-algebra [11, which is a generalization of the superPoincaré algebra]. Although the M-algebraic language is universal (as suggested i.e. by the study of M-brane and D-brane systems), and thus the preon notion is not restricted to considering excitations over the Minkowski vacuum, it is natural to ask ourselves whether preons can be defined in terms of a generalization of the AdS superalgebra. This is tied to the AdS generalization of the M-algebra, which we will call the AdS-M-algebra. Our conclusion, which follows from the BPS preon generalization to be presented here, is that the AdS-M-algebra is to be identified with $\operatorname{osp}(1 \mid 32)$, which in our preonic context appears as a deformation of the M-algebra. The algebra $\operatorname{osp}(1 \mid 32)$ as a generalized AdS superalgebra in $D=11$ had been proposed in [33, 34, 35. (see also [36, 37, 38, 39] for other related superalgebras). The osp(1|32) algebra had already been singled out in the original $D=11$ supergravity paper [10], and used as a basis for a discussion of the gauge structure of $D=11$ supergravity [40, 41] as well as in early discussions of general supersymmetry algebras [42]; its relevance in M(atrix)-theory had been put forward in [43].

\footnotetext{
${ }^{2} \mathrm{~A}$ very recent paper $[21$ states that the maximal fraction $(\neq 32 / 32)$ of supersymmetries preserved by a solution of the (again, free and classical) type IIB supergravity is $28 / 32$.

${ }^{3}$ Let us also note that the above no-go statements have always been made for purely bosonic supersymmetric solutions i.e., for supergravity configurations with all fermionic fields equal to zero, a restriction not implied by the preon conjecture.

${ }^{4}$ To be more precise, this generalization of the superPoincaré algebra is given by the semidirect sum of the M-algebra [11 and $s o(1,10)$ (alternatively, one may take $G L(32, \mathbb{R})$, the M-algebra automorphism group [30, when no decomposition in gamma matrices is assumed), which can be shown to be an expansion [31] of the $\operatorname{osp}(1 \mid 32)$ superalgebra. The (32+528)-dimensional M-algebra itself, which is the maximal central extension of the abelian $\{Q, Q\}=0$ superalgebra of the 32 fermionic generators (see 32]), is a contraction of $\operatorname{ssp}(1 \mid 32)$. Such a contraction is possible because the M-algebra and $\operatorname{ssp}(1 \mid 32)$ have the same dimension.
} 


\section{BPS preons, the preonic supermultiplet and the M-algebra}

An abstract BPS preonic state may be characterized by a single bosonic preonic spinor $\lambda_{\alpha}$,

$$
\mid \text { BPS preon }>=\mid \lambda>\text {, }
$$

which is orthogonal to the 31 bosonic spinors $\epsilon_{I}{ }^{\alpha}, \epsilon_{I}{ }^{\alpha} \lambda_{\alpha}=0$, which determine the 31 supersymmetries preserved by the preon,

$$
\epsilon_{I}^{\alpha} Q_{\alpha} \mid \text { BPS preon }>=0, \quad I=1, \ldots, 31,
$$

(cf. eq. (4) ). Due to the above orthogonality, eq. (6) implies that $Q_{\alpha} \mid \lambda>\propto \lambda_{\alpha}$. This may be expressed as

$$
Q_{\alpha}\left|\lambda>=\lambda_{\alpha}\right| \lambda^{f}>
$$

where $\mid \lambda^{f}>$ is a state with odd Grassmann parity (assuming that the original preonic state $|\lambda\rangle$ is bosonic, as befits a state corresponding to a purely bosonic solution of supergravity. The simplest preonic supermultiplet contains only two states, $\mid \lambda>$ and $\left|\lambda^{f}\right\rangle$,

$$
\| \lambda^{\text {super }}>>:=\left(\begin{array}{l}
\mid \lambda> \\
\mid \lambda^{f}>
\end{array}\right)
$$

with the action of the supersymmetry generator on $\mid \lambda^{f}>$ being defined in terms of the same bosonic spinor $\lambda_{\alpha}$,

$$
Q_{\alpha}\left|\lambda>=\lambda_{\alpha}\right| \lambda^{f}>, \quad Q_{\alpha}\left|\lambda^{f}>=\lambda_{\alpha}\right| \lambda>.
$$

These supersymmetry transformations may be collected in one compact equation

$$
Q_{\alpha}\left\|\lambda^{\text {super }}>>=\chi \lambda_{\alpha}\right\| \lambda^{\text {super }}>>, \quad \chi \chi=1 \quad,
$$

in terms of the preonic supermultiplet $\| \lambda^{\text {super }}>>$ and a Clifford algebra variable $\chi$. When the preonic supermultiplet is represented by a column vector, as in eq. (8), $\chi$ is realized as the $\sigma^{1}$ Pauli matrix, $\chi=\left(\begin{array}{ll}0 & 1 \\ 1 & 0\end{array}\right)$.

Now, assuming that $\lambda_{\alpha}$ is a $c$-number,

$$
Q_{\beta} \lambda_{\alpha}=\lambda_{\alpha} Q_{\beta}
$$

we conclude that the supersymmetry transformations generate the M-algebra,

$$
\left\{Q_{\alpha}, Q_{\beta}\right\}=P_{\alpha \beta}, \quad\left[P_{\alpha \beta}, Q_{\gamma}\right]=0, \quad\left[P_{\alpha \beta}, P_{\gamma \delta}\right]=0 .
$$

Indeed, using (11) we find from (9) that both the BPS preon and its superpartner are eignestates of the generalized momentum $P_{\alpha \beta}$ (here characterized as the most general r.h.s. for the $\left\{Q_{\alpha}, Q_{\beta}\right\}$ anticommutator). The common eigenvalue matrix of $\mid \lambda>$ and $\mid \lambda^{f}>$ is given by the tensor product $\lambda_{\alpha} \lambda_{\beta}$ of two copies of $\lambda$,

$$
\left\{\begin{array}{l}
P_{\alpha \beta}\left|\lambda>=\lambda_{\alpha} \lambda_{\beta}\right| \lambda> \\
P_{\alpha \beta}\left|\lambda^{f}>=\lambda_{\alpha} \lambda_{\beta}\right| \lambda^{f}>
\end{array} \quad \Leftrightarrow \quad P_{\alpha \beta} \| \lambda^{\text {super }}>>=\lambda_{\alpha} \lambda_{\beta}|| \lambda^{\text {super }}>>.\right.
$$

As the preonic spinor $\lambda_{\alpha}$ is a $c$-number (eq. (11) also implies $P_{\alpha \beta} \lambda_{\gamma}=\lambda_{\gamma} P_{\alpha \beta}$ ), one easily finds that on a preonic state or on the preonic supermultiplet $[P, P] \| \lambda>>=0$. This implies $[P, P]=0$ if we do not allow for the presence of other generators, since the possibility $[P, P]=c P$, allowed by Grassmann parity conservation, is ruled out because $\lambda$ is nonvanishing and $[P, P] \mid \lambda>=$ $c \lambda \lambda \mid \lambda>=0$ would require $c=0$. 


\section{The AdS-M-algebra as suggested by AdS preons}

The previous discussion shows that the original definition of the BPS preon [1] reproduces the Malgebra (12), which generalizes the superPoincaré algebra by involving the generalized momenta generator $P_{\alpha \beta}=P_{\beta \alpha}$. This includes, in addition to the standard momenta generator $P_{m}$ (through $\left.P_{\alpha \beta}=P_{m} \Gamma_{\alpha \beta}^{m}\right)$, a set of tensorial central charges that reflect the existence of extended objects in M-theory: they can be realized as topological charges for various branes [44]. For instance, the $S O(1,10)$-covariant decomposition $P_{\alpha \beta}=\Gamma_{\alpha \beta}^{m} P_{m}+i \Gamma_{\alpha \beta}^{a_{1} a_{2}} Z_{a_{1} a_{2}}+\Gamma_{\alpha \beta}^{a_{1} \ldots a_{5}} Z_{a_{1} \ldots a_{5}}$, obtained by using the $D=11$ gamma matrices, includes the two- and five-index central charges $Z_{a_{1} a_{2}}$ and $Z_{a_{1} \ldots a_{5}}$. Their spatial components, $Z_{i_{1} i_{2}}$ and $Z_{i_{1} \ldots i_{5}}$, and those of their duals, $Z^{i_{1} \ldots i_{9}} \propto \epsilon^{0 i_{1} \ldots i_{9} j} Z_{0 j}$ and $Z^{i_{1} \ldots i_{6}} \propto \epsilon^{0 i_{1} \ldots i_{6} j_{1} \ldots j_{4}} Z_{0 j_{1} \ldots j_{4}}$, reflect, respectively, the existence of the M2-brane (elevendimensional supermembrane), the M5-brane, the Horava-Witten hyperplanes (M9-branes) and the Kaluza-Klein monopole (KK6-brane) [44, 45, 46].

To look for the AdS generalization of the BPS preon, we start from the fact that in lower dimensions $D=4,6$ and 10 (with minimal spinor indices $\alpha, \beta=1, \ldots, n$ taking $n=4,8,16$ values respectively). A BPS preon wavefunction in its tensorial coordinate representation given by scalar superfields on the corresponding tensorial superspaces $\Sigma^{\left(\frac{n(n+1)}{2} \mid n\right)}$, can be identified [2, 3, 4] with a wavefunction describing a tower of massless conformal higher spin fields [7, 4] (see Sec. 5$) \sqrt{5}$. Now, the free AdS conformal massless fields can be described in the same manner by the equations for a scalar superfield on the $O S p(1 \mid n)$ supermanifolds which, thus, provide the AdS generalizations of the flat, tensorial $\Sigma^{\left(\frac{n(n+1)}{2} \mid n\right)}$ superspaces [25, 26, 47, 48, 49]. This suggests identifying an AdS preon state with the one whose wavefunction is the $D=11, n=32$ counterpart of the wavefunction describing, in lower $D=4$ and likely in $D=6,10$ dimensions, towers of free conformal higher spin fields in $A d S_{4,6,10}$ spacetimes respectively [26, 49]6.

The first consequence of this assumption is the identification of the AdS-M-algebra. We conclude from the AdS preonic point of view that the appropriate AdS generalization of the Malgebra (see [43, 33, 34, 35, 38] for earlier discussions), the AdS-M-algebra, is the orthosymplectic osp $(1 \mid 32)$ one,

$$
\begin{aligned}
& \left\{Q_{\alpha}, Q_{\beta}\right\}=M_{\alpha \beta}, \quad\left[M_{\alpha \beta}, Q_{\gamma}\right]=\frac{2}{R} C_{\gamma(\alpha} Q_{\beta)}, \\
& {\left[M_{\alpha \beta}, M_{\gamma \delta}\right]=\frac{2}{R}\left(C_{\gamma(\alpha} M_{\beta) \delta}+C_{\delta(\alpha} M_{\beta) \gamma}\right)}
\end{aligned}
$$

where $C_{\alpha \beta}=-C_{\beta \alpha}$ is the nondegenerate $32 \times 32$ invariant $S p(32)$ symplectic metric. The parameter $R$ is introduced to make the possibility of contracting $\operatorname{osp}(1 \mid 32)$ to the M-algebra (12) [43] explicit. It is convenient to take $R$ with dimensions of length; then it corresponds to the radius of the generalized AdS space, for which the $M_{\alpha \beta}$ play the rôle of isometry generators. In the $R \rightarrow \infty$ limit the $M_{\alpha \beta}$ symplectic generators of $\operatorname{osp}(1 \mid 32)$ become the abelian generalized momenta $P_{\alpha \beta}$.. Reciprocally, osp(1|32) is a deformation of the M-algebra characterized by the radius deformation parameter $R$. Algebra contractions abelianize part of the generators, and deformations go in the inverse direction; in view of this, it is not surprising that the AdS preon turns out to be a non-commutative deformation of the original Malgebra preon definition [1].

\footnotetext{
${ }^{5}$ The case $n=2$ corresponds to a scalar superfield on $\Sigma^{(3 \mid 2)}$, which coincides with the standard $D=3$ Minkowski space, and no higher spin fields appear.

6 This is the case for $D=4, n=4$. That a scalar field theory on the $\operatorname{SSp}(1 \mid n)$ supermanifold for $n=8,16$ describes the $D=6,10$ free massless conformal AdS higher spin theories has still to be proven (e.g., by methods similar to those used in [4] to show that a scalar field on the flat $n=8,16$ tensorial spaces describes free conformal higher spin theories in $D=6,10$ Minkowski spaces, respectively).
} 


\section{AdS preons}

The discussion in Sec. 2 indicates that the AdS generalization of the BPS preon notion will require dropping the commutativity property of the preonic spinor since, by assuming eq. (11), we arrived at the M-algebra from the preonic supermultiplet.

Further, since we want that in the $R \rightarrow \infty$ limit the AdS preonic supermultiplet becomes the M-algebra one, we shall assume that the AdS supersymmetry generators transform the AdS preon and its superpartner among themselves in a way similar to (7), where now a noncommuting but still Grassmann even preonic spinor $\Lambda_{\alpha}$ replaces the $c$-number $\lambda_{\alpha}$,

$$
Q_{\alpha}\left|\lambda>=\Lambda_{\alpha}\right| \lambda^{f}>, \quad Q_{\alpha}\left|\lambda^{f}>=\Lambda_{\alpha}\right| \lambda>, \quad\left[\Lambda_{\alpha}, \Lambda_{\beta}\right] \neq 0 .
$$

To have a suitable $R \rightarrow \infty$ limit, we conclude that $\left[\Lambda_{\alpha}, \Lambda_{\beta}\right] \propto \frac{1}{R}$. As the required coefficient is a dimensionless antisymmetric spin-tensor, it is natural to identify it with $C_{\alpha \beta}$. In such a way we find the following commutation relations for the $\Lambda_{\alpha}$ spinor operator entering (15),

$$
\left[\Lambda_{\alpha}, \Lambda_{\beta}\right]=-\frac{i}{2 R} C_{\alpha \beta}
$$

which can be realized by

$$
\Lambda_{\alpha}=\lambda_{\alpha}-\frac{i}{4 R} C_{\alpha \beta} \frac{\partial}{\partial \lambda^{\beta}}
$$

Notice that the replacement $\lambda_{\alpha} \rightarrow \Lambda_{\alpha}$ can be treated as passing to the Moyal star product,

$$
\lambda_{\alpha} \cdot \rightarrow \Lambda_{\alpha} \cdot=\lambda_{\alpha} *
$$

see [26]. Eqs. (16), (18) are a deformation of the abelian $\left[\lambda_{\alpha}, \lambda_{\beta}\right]=0$, and so eqs. (15), (16) constitute a deformation of (10) resulting from the non-commutativity of $\Lambda_{\alpha}$. In the $R \rightarrow \infty$ limit of the deformation parameter, $\Lambda_{\alpha}$ becomes the commutative preonic spinor $\lambda_{\alpha}$ of the previous 'flat' case. Thus, the flat limit of the AdS preon reproduces the original M-algebraic BPS preon definition [1, of which the AdS preon is a deformation.

Denoting the AdS preonic supermultiplet also by $\| \lambda^{\text {super }}>>$, as in eq. (8), the two equations in eq. (15) are collected in a single equation $(c f .(10)$ ),

$$
Q_{\alpha}\left\|\lambda^{\text {super }}>>=\chi \Lambda_{\alpha}\right\| \lambda^{\text {super }}>>, \quad \chi \chi=1, \quad \Lambda_{\alpha}=\lambda_{\alpha}-\frac{i}{4 R} C_{\alpha \beta} \frac{\partial}{\partial \lambda^{\beta}},
$$

which involves the Clifford algebra element $\chi$ (see eq. (10) ) and the non-commutative preonic spinor $\Lambda_{\alpha}$. Thus, the AdS preonic supermultiplet is associated with the following representation of the generators of the $\operatorname{osp}(1 \mid 32)$ algebra (eq. (14))

$$
Q_{\alpha}=\chi \Lambda_{\alpha}, \quad M_{\alpha \beta}=2 \Lambda_{(\alpha} \Lambda_{\beta)},
$$

where $\Lambda_{\alpha}$ satisfies the algebra of eq. (16) and $\chi^{2}=1$. In matrix form, the preonic realization of the $\operatorname{osp}(1 \mid 32)$ generators reads

$$
Q_{\alpha}=\left(\begin{array}{cc}
0 & \Lambda_{\alpha} \\
\Lambda_{\alpha} & 0
\end{array}\right), \quad M_{\alpha \beta}=\left(\begin{array}{cc}
2 \Lambda_{(\alpha} \Lambda_{\beta)} & 0 \\
0 & 2 \Lambda_{(\alpha} \Lambda_{\beta)}
\end{array}\right) .
$$

The basic commutation relations of $\Lambda_{\alpha}$ together with the representation of $M_{\alpha \beta}$ in (20) are collected in the multiplication table

$$
\Lambda_{\alpha} \Lambda_{\beta}=-\frac{i}{4 R} C_{\alpha \beta}+\frac{1}{2} M_{\alpha \beta}
$$




\section{BPS preons, tensorial superspaces and massless conformal higher spin fields}

Our AdS generalization of the M-algebraic BPS preon, eq. (15), and its associated AdSM-algebra, are suggested by the properties of higher spin theory as described by scalar superfields in tensorial superspaces. This will be shown in this section, which we begin by considering the realization of the M-algebra preon as a scalar superfield in flat, tensorial superspace before moving to the AdS case in Sec. 5.5.

\subsection{Preonic superwavefunction in tensorial superspace $\sum^{\left(\frac{n(n+1)}{2} \mid n\right)}$}

Tensorial superspaces $\Sigma^{\left(\frac{n(n+1)}{2} \mid n\right)}$ are parametrized by $n(n+1) / 2$ even spin-tensor coordinates $X^{\alpha \beta}$ and by $n$ odd, fermionic coordinates $\theta^{\alpha}$ (see e.g. [8, 12, 32, 3]),

$$
\Sigma^{\left(\frac{n(n+1)}{2} \mid n\right)}=\left\{\left(X^{\alpha \beta}, \theta^{\alpha}\right)\right\}, \quad X^{\alpha \beta}=X^{\beta \alpha}, \quad \alpha=1, \ldots, n .
$$

In $D=4,10$ and 11 the minimal spinors have $n=4,16$ and 32 components, and their associated even coordinates $X^{\alpha \beta}$ have 10, 136 and 528 components respectively. These include, besides those of the spacetime $D$-vector, additional bosonic tensorial coordinates. Specifically,

$$
\begin{array}{cl}
D=4: & \Sigma^{(10 \mid 4)}=\left\{\left(x^{m}, y^{[m n]}, \theta^{\alpha}\right)\right\}, \quad X^{\alpha \beta}=x^{m} \gamma_{m}^{\alpha \beta}+y^{m n} \gamma_{m n}^{\alpha \beta} ; \\
D=10: & \Sigma^{(136 \mid 16)}=\left\{\left(x^{m}, y^{[m n p q r]}, \theta^{\alpha}\right)\right\}, \quad X^{\alpha \beta}=x^{m} \tilde{\sigma}_{m}^{\alpha \beta}+y^{m n p q r} \tilde{\sigma}_{m n p q r}^{\alpha \beta} \\
D=11: & \Sigma^{(528 \mid 32)}=\left\{\left(x^{m}, y^{[m n]}, y^{[m n p q r]}, \theta^{\alpha}\right)\right\} \\
& X^{\alpha \beta}=x^{m} \Gamma_{m}^{\alpha \beta}+y^{m n} \Gamma_{m n}^{\alpha \beta}+y^{m n p q r} \Gamma_{m n p q r}^{\alpha \beta} .
\end{array}
$$

The generalized momentum and the supersymmetry generators can be realized as differential operators in $\Sigma^{\left(\frac{n(n+1)}{2} \mid n\right)}$,

$$
P_{\alpha \beta}=-i \partial_{\alpha \beta}, \quad Q_{\alpha}=\partial_{\alpha}-i \theta^{\beta} \partial_{\alpha \beta}, \quad \text { where } \quad \partial_{\alpha \beta}:=\frac{\partial}{\partial X^{\alpha \beta}}, \quad \partial_{\alpha}:=\frac{\partial}{\partial \theta^{\alpha}}
$$

(these give $\left\{Q_{\alpha}, Q_{\beta}\right\}=2 P_{\alpha \beta}$, but the inclusion of the 2 here simplifies the coefficients below). The $\left(X^{\alpha \beta}, \theta^{\alpha}\right)$ coordinates representation of the BPS preonic supermultiplet $\| \lambda^{\text {super }}>>$ wavefunction is

$$
\Phi_{(\lambda, \chi)}(X, \theta)=<<X, \theta \| \lambda^{\text {super }}>>\quad ;
$$

notice that the $\chi$ dependence of the l.h.s. comes from $\left.\| \lambda^{\text {super }}\right\rangle>$, see eq. (19).

The defining relation (13) implies that $\Phi$ satisfies the differential superwave equation

$$
\left(\partial_{\alpha \beta}-i \lambda_{\alpha} \lambda_{\beta}\right) \Phi_{(\lambda, \chi)}(X, \theta)=0
$$

This preonic equation [3] coincides with the unfolded equations for higher spin fields [47, 28] formulated in tensorial space 7 ; it appeared for the first time in the quantization [7] of the generalized superparticle model [8] on tensorial superspace $\Sigma^{\left(\frac{n(n+1)}{2} \mid n\right)}$.

\footnotetext{
${ }^{7}$ In refs. [47, 48, 28, the unfolded equations are written in the form $\left(\frac{\partial}{\partial X^{\alpha \beta}}-i \frac{\partial}{\partial \mu^{\alpha}} \frac{\partial}{\partial \mu^{\beta}}\right) C(X, \mu)=0$, which is related to the preonic equation (29) by a Fourier transformation in the auxiliary bosonic spinor variable $\lambda_{\alpha}$.
} 


\subsection{A model for a pointlike BPS preon in tensorial superspace $\sum^{\left(\frac{n(n+1)}{2} \mid n\right)}$}

The action for a superparticle in $\Sigma^{\left(\frac{n(n+1)}{2} \mid n\right)}$ with one auxiliary bosonic spinor reads [8]

$$
S=\int d \tau \lambda_{\alpha} \lambda_{\beta}\left(\dot{X}^{\alpha \beta}-i \dot{\theta}^{(\alpha} \theta^{\beta)}\right) \quad, \quad \alpha=1, \ldots, n \quad .
$$

It describes a 0-brane preon [2, 3] or preonic superparticle since its ground state preserves $(n-1)$ out of $n$ supersymmetries. The $\Sigma^{\left(\frac{n(n+1)}{2} \mid n\right)}$ superspace preonic wavefunction is obtained from the quantization of the 0-brane model (30). To exhibit this schematically let us note that eqs. (13), (29) look as the quantum mechanical representation of the generalized Cartan-Penrose relation

$$
P_{\alpha \beta}-\lambda_{\alpha} \lambda_{\beta} \approx 0,
$$

which appears as a primary constraint for the canonical generalized momentum for $X^{\alpha \beta}$. Actually, the situation is slightly more complicated, because this constraint is not first class, and its conversion to a first class constraint requires the addition of a new variable. We will just state the results and refer to [7] for details.

The quantization of the pointlike preon model (30) produces a superwavefunction $\Upsilon$ that depends on $X^{\alpha \beta}, \theta^{\alpha}, \lambda_{\alpha}$, and on an additional Clifford algebra variable $\tilde{\chi}, \tilde{\chi}^{2}=1$, which is introduced in the process of converting the fermionic second class constraint into a first class one. The wavefunction $\Upsilon$ satisfies the wave equation [7] which results from imposing the 32 fermionic first class constraints of the converted system,

$$
\left(D_{\alpha}-\tilde{\chi} \lambda_{\alpha}\right) \Upsilon(X, \theta, \lambda, \tilde{\chi})=0, \quad D_{\alpha}:=\partial_{\alpha}+i \theta^{\beta} \partial_{\alpha \beta}, \quad \tilde{\chi}^{2}=1,
$$

where $D_{\alpha}$ is the covariant derivative in tensorial superspace commuting with the supersymmetry generator $Q_{\beta}$ in (27). Thus, eq. (32) is supersymmetry invariant provided that $\tilde{\chi}$ is inert under supersymmetry (as the bosonic spinor variable $\lambda_{\alpha}$ is). The consistency conditions for the quantum fermionic first class constraints (32) give the bosonic first class constraint

$$
\left(\partial_{\alpha \beta}-i \lambda_{\alpha} \lambda_{\beta}\right) \Upsilon(X, \theta, \lambda, \tilde{\chi})=0,
$$

a clear counterpart of (29)).

Although (32) is similar to (101), it includes the supersymmetric covariant derivatives $D_{\alpha}$ rather than $Q_{\alpha}$ in (27). To solve this, let us now observe that the shift of a Clifford algebra variable $\chi$ by a nilpotent one $\psi, \chi \rightarrow \tilde{\chi}=\chi-\psi$, is still a Clifford element if the shift anticommutes with $\chi,\{\chi, \psi\}=0$. In the present case, and with $\tilde{\chi}=\chi-2 \theta \lambda$, we find

$$
(\chi-2 \theta \lambda)^{2}=1 \quad \Leftarrow \quad\left\{\begin{array}{c}
\chi^{2}=1, \quad(\theta \lambda)^{2}=0, \\
\{\chi, \theta \lambda\}=0 .
\end{array}\right.
$$

With this in mind it is easily seen that eq. (32) gives the coordinate representation of the transformation rules (10), $\left(Q_{\alpha}-\chi \lambda_{\alpha}\right) \Upsilon=0$, of the preonic supermultiplet provided we identify

$$
\Upsilon(X, \theta, \lambda, \tilde{\chi})=\Phi_{(\lambda, \chi)}(X, \theta):=<<X, \theta \| \lambda^{\text {super }}>>, \quad \tilde{\chi}=\chi-2 \theta \lambda .
$$

For $n=4,8,16(D=4,6,10)$ the above wavefunction, with the additional projection condition $\Upsilon(X, \theta, \lambda, \tilde{\chi})=\Upsilon(X, \theta,-\lambda,-\tilde{\chi})$ (see [7, 49] for a discussion), describes a tower of massless, conformal higher spin fields [7, 4]. 


\section{3 $\mathrm{D}=4,6,10$ massless conformal higher spin fields from the pre- onic wavefunction on $\Sigma^{\left(\frac{n(n+1)}{2} \mid n\right)}(n=4,8,16)$}

A Clifford superfield [50] is a function depending on Clifford algebra variables, like our $\Upsilon(\tilde{\chi})$ in (35) with $\tilde{\chi} \tilde{\chi}=1$. It is similar to the familiar superfields in that its series decomposition in the Cifford algebra arguments is finite. In the present case, where $\Upsilon(\tilde{\chi})$ depends on only one Clifford variable, the superfield contains only two (superfield) components,

$$
\Upsilon(X, \theta, \lambda, \tilde{\chi})=\Phi^{0}(X, \theta, \lambda)+\tilde{\chi} \Phi^{1}(X, \theta, \lambda)
$$

Eq. (32) implies that

$$
D_{\alpha} \Phi^{0}=\lambda_{\alpha} \Phi^{1} \quad, \quad D_{\alpha} \Phi^{1}=\lambda_{\alpha} \Phi^{0} \quad
$$

These equations can be solved by expressing, say, $\Phi^{1}$ in terms of $\Phi^{0}$, although to write such an expression in a $G L(n)$-covariant manner one has to introduce a bosonic spinor $u^{\alpha}$ 'dual' to $\lambda_{\alpha}$ (i.e. $\left.u^{\alpha} \lambda_{\alpha}=1\right): \Phi^{1}=-i u^{\alpha} D_{\alpha} \Phi^{0}$. Now applying $D_{\beta}$ to the first equation in (37) and using the second one, we find the following equation restricting only the $\Phi^{0}(X, \theta, \lambda)$ superfield (see [49])):

$$
\left(D_{\alpha} D_{\beta}-\lambda_{\alpha} \lambda_{\beta}\right) \Phi^{0}=0
$$

The symmetric part of (47) gives the preonic equation (29), while the antisymmetric part reads $D_{[\alpha} D_{\beta]} \Phi^{0}=0$. This equation was proposed in [49] as a superfield generalization of the Vasiliev field equations [47, 28] for the wavefunctions describing the towers of all the bosonic and fermionic conformal higher spin fields in $D=4$ tensorial space. Indeed, the same equation is obeyed by the wavefunction integrated over the bosonic spinor space $\phi(X, \theta)=\int d^{n} \lambda \Phi^{0}(X, \theta, \lambda)$,

$$
D_{[\alpha} D_{\beta]} \phi(X, \theta)=0 .
$$

Inserting the superfield expansion

$$
\phi(X, \theta)=b(X)+\theta^{\alpha} f_{\alpha}(X)+\sum_{i=2}^{n} \theta^{\alpha_{1}} \ldots \theta^{\alpha_{i}} \phi_{\alpha_{1} \ldots \alpha_{i}}(X),
$$

in eq. (39), one finds [49] that the higher components of the $\phi(X, \theta)$ superfield vanish, $\phi_{\alpha_{1} \ldots \alpha_{i}}(X)=0$ for $i \geq 2$, and that the first two obey the bosonic and fermionic Vasiliev equations [47]

$$
\partial_{\alpha[\beta} \partial_{\gamma] \delta} b(X)=0, \quad \partial_{\alpha[\beta} f_{\gamma]}(X)=0, \quad \alpha, \beta, \gamma, \delta=1, \ldots, n .
$$

The proof that for $n=4$ these equations give a tower of all the $D=4$ massless higher spin fields was given in [47]. That the $n=8$ and $n=16$ equations also describe a tower of conformal massless fields in $D=6$ and $D=10$ was shown in [4], to which we refer the reader for details. 


\subsection{Continuous spectrum of the $\mathrm{D}=11$ preonic superparticle}

The situation for the $D=11, n=32 \mathrm{M}$-theoretic case is less clear. What makes it different from the previous $D=3,4,6$ and 10 cases is that in $D=11$ the vector $\lambda \Gamma_{m} \lambda$ is not lightlike, $\left(\lambda \Gamma_{m} \lambda\right)^{2} \neq 0$, which means that $P_{m} P^{m} \neq 0$ for the $D=11$ spacetime momentum $P_{m}=\Gamma_{m}^{\alpha \beta} P_{\alpha \beta} \propto \lambda \Gamma_{m} \lambda$. Moreover, $P_{m} P^{m}$ becomes an arbitrary constant for the tensorial superspace $\Sigma^{(528 \mid 32)}$ pointlike preon model of eq. (30) [ [8, 12], which is said to have a 'dynamically generated mass' [51]. This property is tantamount to having a continuous mass spectrum. Since this is typical of a composite system, we arrive at a complementary description of a BPS preon: albeit fundamental, it possesses a property associated with composite systems. This situation is not new: the $D=11$ supermembrane (M2-brane) was considered, as a fundamental object, as a $D=11$ counterpart of the $D=10$ fundamental string and, at the same time, it was shown to have a continuous spectrum, a property that was explained in the Matrix model conjecture in which the M2 brane is considered as a composite of D0-branes ( $\mathrm{N}=2, D=10$ massive superparticles). Such a D0-brane picture is dual to the one in which the M2-brane is considered to be fundamental. As for preons, we also have that an elementary preon state has components in all the tensorial charges associated with the $1 / 2$ BPS branes, which are themselves composite in the preonic picture. We note, however, this latter property is also shared with the $D=4,6,10$ dimensional counterparts of the M-algebraic BPS preon, which nevertheless do not possess a continuous mass spectrum and rather describe towers of massless conformal higher spin fields as already discussed. The above dual aspect of the preon holds for $D=11$, the M-theory dimension.

The mechanism to construct of $k / 32$-BPS states with $1<k<31$ from the BPS preons is unknown, and one of the motivations to study further the properties of BPS preons is to look for new insights in this direction. It is natural to assume that the reduced supersymmetry of a $k / 32$-BPS state containing $\tilde{n}=32-k$ preons is the result of some kind of 'interaction' among them. If so, a possible description of such an interaction in $D=11$ should be similar to a theory of interacting higher spin fields in the lower $D=4,6,10$ dimensions. It is known that a selfconsistent interaction of higher spin fields is possible in AdS but not in Minkowski spacetime [5] (the interaction depends on the inverse of the cosmological constant [52, 5]). Thus, the search for a selfconsistent interaction of an infinite tower of higher spin fields begins by formulating the free equations for these fields in AdS spacetime or an AdS superspace.

\subsection{Equations for $A d S_{4}$ conformal higher spin fields on the $O S p(1 \mid 4)$ supergroup manifold}

The AdS generalization of the free higher spin equations in tensorial superspace, eq.(32), was obtained in [26]. In our notation it reads [49]

$$
\left(\nabla_{\alpha}-\tilde{\chi} \Lambda_{\alpha}\right) \Upsilon(X, \theta, \lambda, \tilde{\chi})=0, \quad \Lambda_{\alpha}=\lambda_{\alpha}-\frac{1}{4 R} C_{\alpha \beta} \frac{\partial}{\partial \lambda^{\beta}}, \quad \tilde{\chi}^{2}=1, \quad \alpha=1, \ldots, n,
$$

where $\nabla_{\alpha}$ is defined by the decomposition of the exterior derivative acting on the $O S p(1 \mid n)$ manifold,

$$
d=E^{\alpha \beta} \nabla_{\alpha \beta}+E^{\alpha} \nabla_{\alpha}
$$


in terms of the left-invariant Maurer-Cartan (MC) forms $\left(E^{\alpha \beta}, E^{\alpha}\right)$. These satisfy the $\operatorname{osp}(1 \mid n) \mathrm{MC}$ equations,

$$
d E^{\alpha \beta}+\frac{1}{R} C_{\gamma \delta} E^{\alpha \gamma} \wedge E^{\beta \delta}+i E^{\alpha} \wedge E^{\beta}=0 \quad, \quad \mathcal{D} E^{\alpha}:=d E^{\alpha}+\frac{1}{R} C_{\gamma \delta} E^{\alpha \gamma} \wedge E^{\delta}=0 .
$$

The above $\nabla_{\alpha}$ and $\nabla_{\alpha \beta}$ satisfy the $\operatorname{osp}(1 \mid n)$ superalgebra,

$$
\begin{gathered}
\left\{\nabla_{\alpha}, \nabla_{\beta}\right\}=2 i \nabla_{\alpha \beta} \quad, \quad\left[\nabla_{\alpha \alpha^{\prime}}, \nabla_{\beta}\right]=\frac{2 i}{R} C_{\beta(\alpha} \nabla_{\left.\alpha^{\prime}\right)} \\
{\left[\nabla_{\alpha \beta}, \nabla_{\gamma \delta}\right]=\frac{2 i}{R} C_{\alpha(\gamma} \nabla_{\delta) \beta}+\frac{2 i}{R} C_{\beta(\gamma} \nabla_{\delta) \alpha}}
\end{gathered}
$$

Decomposing the Clifford superfield $\Upsilon$ (eq. (36) ), it is found that its second component can be expressed in terms of the first one (as in (37) for flat tensorial superspace) and that its first component obeys [49]

$$
\left(\nabla_{\alpha} \nabla_{\beta}+\Lambda_{\beta} \Lambda_{\alpha}\right) \Phi^{0}=0
$$

The symmetric $(\alpha \beta)$ part of this equation gives [27] the AdS preonic equation that generalizes (33),

$$
\left(\nabla_{\alpha \beta}-\frac{i}{2}\left(\Lambda_{\alpha} \Lambda_{\beta}+\Lambda_{\beta} \Lambda_{\alpha}\right)\right) \Upsilon(X, \theta, \lambda, \tilde{\chi})=0 .
$$

The antisymmetric $[\alpha \beta]$ part of (47) gives the AdS generalization of equation (39) for a scalar superfield in flat tensorial superspace proposed in [49],

$$
\left(\nabla_{[\alpha} \nabla_{\beta]}+\frac{i}{4 R} C_{\alpha \beta}\right) \Phi(X, \theta)=0,
$$

The set of Eqs. (42) and (48) is equivalent to the following one-form differential equation proposed in [26]

$$
\left(d-\hat{w}_{0}\right) \hat{\Upsilon}=0
$$

where $\hat{w}_{0}$ is given by $\hat{w}_{0}=E^{\alpha \beta} M_{\alpha \beta}+E^{\alpha} Q_{\alpha}$ with $\left.M_{\alpha \beta}=2 \Lambda_{(\alpha} \Lambda_{\beta}\right), Q_{\alpha}=\chi \Lambda_{\alpha}$ (eqs. (20)) and $\Lambda_{\alpha}$ obeys the commutation relations (16)..$\hat{\Upsilon}$ depends on the $\left(X^{\alpha \beta}, \theta^{\alpha}\right)$ variables of the $O S p$ supergroup manifold, as well as on $\tilde{\chi}$ and the operator $\Lambda$, which is why $\hat{\Upsilon}$ (denoted $\mid \Phi>$ ) was called Fock module in [26]. Eq. (50) can also be written in the form [26]

$$
\left(d-w_{0} *\right) \Upsilon=0
$$

where $w_{0}=E^{\alpha \beta} \lambda_{\alpha} \lambda_{\beta}+E^{\alpha} \chi \lambda_{\alpha}$ is now used with the star product of Eq. (18). The selfconsistency equations for (51), $d w_{0}=w_{0} * \wedge w_{0}$, give the osp MC equations (44). The same equation without star product, $\left(d-w_{0}\right) \Upsilon=0$, leading through it selfconsistency conditions to the MC equations of the tensorial superspace algebra, describes free higher spin fields in flat Minkowski spacetime. Thus, the transition from the Minkowski higher spin field equations in flat tensorial superspace to the equations on the $O S p$ supergroup manifold describing the higher spin fields in $A d S_{4}$ is given by a deformation which introduces non-commutativity (see [24]).

Summarizing, the AdS preon of Sec. 4 can be described by the scalar field theory on the $O S p(1 \mid 32)$ supergroup manifold. This is the $n=32(D=11)$ element of a family of scalar field theories on the $O S p(1 \mid n)$ manifolds, the $n=4$ representative of which, $O S p(1 \mid 4)$, describes the higher spin theory on $A d S_{4}$. As for the $n=8$ and $n=16$ cases, $O S p(1 \mid 8)$ and $O S p(1 \mid 16)$, they are likely to describe the corresponding massless conformal higher spin theories on $A d S_{6}$ and $A d S_{10}$ spaces (see footnote [6). 


\section{The AdS preon as a BPS state. Preservation of all but one AdS supersymmetries.}

The preonic spinors $\Lambda_{\alpha}$ of the AdS preon are non-commuting (eqs. (15) and (19)) and so are $M_{\alpha \beta}$ in $\operatorname{osp}(1 \mid 32)$ (14) that replace the commutative $P_{\alpha \beta}$ of the M-algebra (12). As a result, the 'momenta' sector of the $\operatorname{osp}(1 \mid n)$ superalgebra does not allow for the M-algebraic analysis in [1] and it is not obvious how to relate our AdS preon with the preservation of a fraction of the supersymmetries, a typical property of a BPS state.

To clarify this point, let us use the fact [27] that the scalar superfield equations on the $O S p(1 \mid n)$ supergroup manifold, eqs. (42), (48), appear in the quantization of the generalized superparticle on the $\operatorname{OSp}(1 \mid n)$ supermanifold 8 [25]

$$
S=\int d \tau \lambda_{\alpha} \lambda_{\beta} \hat{E}_{\tau}^{\alpha \beta}
$$

where $\hat{E}_{\tau}^{\alpha \beta} d \tau$ is the pullback to the worldline of the $E^{\alpha \beta}$ Maurer-Cartan form on OSp (see eq.(44), $c f$. 30). This superparticle has the properties of an AdS preon: its ground states preserve all the supersymmetries but one, as reflected by the $31 \kappa$-symmetries possessed by the $n=32$ version of the $O S p(1 \mid n)$ model of Eqs. (52) (see [2, 3] for further discussion in the M-algebraic language).

To clarify this point, consider first the case of a pointlike M-algebra preon. The preonic 0 -brane action in flat tensorial superspace is given by eq. (30). A preonic BPS state can be associated with a purely bosonic solution of the equations of motion that follow from this action. This is preserved by the supersymmetries which keep the fermionic field equal to zero, $\theta(\tau)=0$. The complete set of fermionic symmetries of the action (30) include global supersymmetry $\varepsilon$ and local fermionic $\kappa$-symmetry. In the present case of flat tensorial superspace, a general fermionic transformation $\delta=\delta_{\varepsilon}+\delta_{\kappa}$ reads [8]

$$
\delta \theta^{\alpha}=\delta_{\varepsilon} \theta^{\alpha}+\delta_{\kappa} \theta^{\alpha}:=\varepsilon^{\alpha}+\kappa^{I}(\tau) \epsilon_{I}^{\alpha}(\tau), \quad \epsilon_{I}(\tau)^{\alpha} \lambda_{\alpha}(\tau)=0, \quad \begin{aligned}
& I=1, \ldots, 31 \\
& \alpha=1, \ldots, 32
\end{aligned}
$$

where the 31 bosonic spinors $\epsilon_{I}^{\alpha}(\tau)$ are defined by the condition of being orthogonal to $\lambda_{\alpha}(\tau)$. Then the supersymmetry which is preserved by the purely bosonic, $\theta^{\alpha}(\tau)=0$ ground state solution is characterized by

$$
\varepsilon^{\alpha}=-\kappa^{I} \epsilon_{I}^{\alpha} \quad \Leftarrow \quad \delta \theta^{\alpha}=0
$$

This supersymmetry depends on the 31 parameters $\kappa^{I}$ of local fermionic $\kappa$-symmetry, which become constant on the solution. The fermionic spinor $\varepsilon^{\alpha}$ is constant and so

\footnotetext{
8 The $O S p(1 \mid n)$ supergroup manifold is ' $G L(n)$ flat' [27] and this allows to relate the AdS and the flat tangent superspace versions of the generalized superparticle or preonic 0-brane model of 8 , (Eqs. (52), (30) ). After a Penrose twistor transform in both of them, they are described by the same action in terms of a real $O S p$ supertwistor [8] (fundamental representation of $O S p(1 \mid 2 n)$ ). Then, the quantization in momentum space gives the same wavefunctions, and the specific AdS or Minkowski spacetime wavefunctions are obtained by defining appropriate measures for the Fourier transforms that lead to their coordinate representation [27. This is related to the fact that both the flat tensorial superspace $\Sigma^{\left(\frac{n(n+1)}{2} \mid n\right)}$ and the $O S p(1 \mid n)$ supergroup manifolds can be identified with different cosets $O S p(1 \mid 2 n) /\left[G L(n) \otimes \Sigma^{\left(\frac{n(n+1)}{2} \mid n\right)}\right]$ of the $\operatorname{OSp}(1 \mid 2 n)$ supergroup with respect to differently chosen tensorial superspace subgroups $\check{\Sigma}^{\left(\frac{(n(n+1))}{2} \mid n\right)}$ in $O S p(1 \mid 2 n)$.
} 
should be $\kappa^{I} \epsilon_{I}^{\alpha}$; the rôle of the equations of motion in the supersymmetry preservation is seen at this point. Indeed, the auxiliary bosonic spinor $\lambda_{\alpha}$ is constant on-shell, $\partial_{\tau} \lambda_{\alpha}=0$, so that the 31 bosonic spinors $\epsilon_{I}^{\alpha}$ orthogonal to it can be chosen constant as well and so $\kappa^{I}$ is also constant (see eq. (54)). Thus, the constant $\varepsilon$ of the preserved rigid supersymmetry $\varepsilon^{\alpha}$ is the sum of products of the odd, constant $\kappa^{I}$ with the constant bosonic spinors $\epsilon_{I}{ }^{\alpha}$. The presence of 31 free fermionic parameters $\kappa^{I}$ in (54) allows us to state that a bosonic solution of the equations following from the action (30) preserves 31 target space supersymmetries. This property allows us to identify [2, 3] the ground state of the 0-brane model (30) with a pointlike BPS preon, as it preserves 31 out of the 32 supersymmetries. Further, this preservation of the target supersymmetries (tensorial superspace or M-algebra supersymmetries) may be formulated in abstract quantum mechanical terms for a BPS preon state, as in eq. ([6), without reference to any specific coordinates or momenta representation.

The situation is different in the AdS case. An AdS preon appears as bosonic solution of the equations of motion that follow from the action (52) on the $O S p(1 \mid 32)(O S p(1 \mid n))$ supergroup manifold. The superparticle lagrangian is now given in terms of the bosonic $\mathrm{MC}$ forms of $O S p(1 \mid n)$ (eqs. (43)). The action is again invariant under the 31- $((n-1)-)$ parametric $\kappa$-symmetry transformations characterized by [25]

$i_{\kappa} E^{\alpha \beta}:=\delta_{\kappa} Z^{M} E_{M}^{\alpha \beta}(Z)=0, \quad i_{\kappa} E^{\alpha} \lambda_{\alpha}:=\delta_{\kappa} Z^{M} E_{M}^{\alpha} \lambda_{\alpha}=0, \quad Z^{M}:=\left(X^{\alpha \beta}, \theta^{\alpha}\right)$,

which can be described in terms of $31(n-1)$ bosonic spinors $\epsilon_{I}{ }^{\alpha}$ orthogonal to $\lambda_{\alpha}$ as in eq. (41),

$$
i_{\kappa} E^{\alpha}:=\delta_{\kappa} Z^{M} E_{M}^{\alpha}=\kappa^{I}(\tau) \epsilon_{I}^{\alpha}(\tau) .
$$

The equations of motion for the action (52) include $\mathcal{D}\left(\lambda_{\alpha} \lambda_{\beta}\right)=0$, with the $S p(n)$ covariant derivative $\mathcal{D} \lambda_{\alpha}:=d \lambda_{\alpha}+\frac{1}{R} E_{\alpha}^{*} \lambda_{\beta}$ as in (44). Since $\epsilon_{I}^{\alpha} \lambda_{\alpha}=0$, the bosonic 'Killing' spinors $\epsilon_{I}{ }^{\alpha}$ in (56) are now covariantly constant rather than constant, $\mathcal{D} \epsilon_{I}{ }^{\alpha}:=d \epsilon_{I}{ }^{\alpha}-\frac{1}{R} \epsilon_{I}{ }^{\beta} E_{\beta}^{{ }^{\alpha}}=0$ and hence they are $\tau$-dependent.

The transformation of the fermionic coordinate functions under the $O S p(1 \mid 32)$ symmetry of the action reads $\delta_{\varepsilon} \theta^{\alpha}(\tau)=\varepsilon^{\alpha}-\frac{1}{R} \varepsilon^{\beta} X_{\beta}^{\alpha}(\tau)+O\left(\frac{1}{R^{2}}\right)+O(\theta \theta)$. Thus, the supersymmetries preserved by the ground state with $\theta^{\alpha}=0$ are characterized by ( $c f$. eq. (54) )

$$
\varepsilon^{\alpha}=-\left.\kappa^{I} \epsilon_{I}{ }^{\beta}\left(\delta_{\beta}{ }^{\alpha}+\frac{1}{R} X_{\beta}{ }^{\alpha}+O\left(\frac{1}{R^{2}}\right)\right) \Leftarrow \delta_{\varepsilon} \theta^{\alpha}\right|_{\theta=0}=0
$$

For finite $R$ the terms involving the explicit $X^{\alpha \beta}$ (and $\theta^{\alpha}$ ) dependence hamper the abstract quantum mechanical description of the supersymmetries preserved by the AdS preonic superparticle ground state. When $R \mapsto \infty$, in which limit $\varepsilon^{\alpha}$ becomes constant, eq. (57) reproduces (54) and an abstract quantum mechanical description of the preserved symmetries becomes possible.

Hence, our AdS preon is a BPS state preserving $31((n-1)$ in general) supersymmetries. This can be seen in the generalized coordinates representation of the preonic superwavefunction or through the corresponding pointlike model of eq. (52), where one also observes (eq. (57)) that the preserved supersymmetries are $X$ - (and $\theta$-)dependent. This shows why in the AdS case it is difficult to describe the preserved supersymmetries in an abstract quantum mechanical state terms. In other words, the above discussion explains why representation of the $O S p$ supersymmetry generators on the states in (15), 
which emphasize the single broken supersymmetry, cannot be reformulated through the 31 preserved supersymmetries. Such a representation is provided, instead, by a deformation of the M-algebraic definition of the single supersymmetry broken by the BPS preon. This is obtained by replacing the bosonic spinor $\lambda_{\alpha}$ by the non-commutative preonic spinor $\Lambda_{\alpha}$ (eqs. (15), (16) ) or by moving to the Moyal product, $\lambda_{\alpha} \cdot \mapsto \Lambda_{\alpha}=\lambda_{\alpha} *$, eq. (18).

\section{Conclusions and discussion}

We have given here the AdS generalization of the M-algebraic definition of the BPS preon. Although the M-algebra language is meant to be universal (as suggested by the study of the 1/2-BPS superbrane states), and so is the preon concept [1], the question of its AdS generalization arises naturally when considering a preon as an excitation over a fully supersymmetric AdS-type (rather than Minkowski) vacuum. We have then found that the AdS preon is a deformation of the M-algebra one [1] (as e.g, eq. (19) is a deformation of (10) $)$. This deformation character is exhibited by the explicit presence of $1 / R$ in all the AdS equatios, which reproduce those of the flat case in the $R \rightarrow \infty$ limit. Conversely, all our AdS equations are obtained by the replacement of the $\cdot$ product by the star $*$ one, eq. (18), in the M-algebraic flat ones.

Our generalization is suggested by the observation that the $D=4,6,10$ tensorial superspace counterparts of the M-algebra BPS preon can be identified [2, 3, 49] with the towers of all the free massless, conformal higher spin fields in the respective flat Minkowski spaces. In other words, the wavefunctions of the $n=4$ and $n=8,16$ counterparts of the M-algebra BPS preons in flat tensorial superspaces (the manifolds of the rigid $\Sigma^{\left(\frac{n(n+1)}{2} \mid n\right)}$ tensorial superspace groups) describe infinite towers of free conformal higher spin field strengths in $D=4$ [7] (see also [47]) and in $D=6,10$ [4]. Similarly, we identify the wavefunction of an AdS preon state with the $O S p(1 \mid 32)$ counterpart of the scalar superfield on the $O S p(1 \mid 4)$ supergroup manifold which describes [26, 27, 49] all the conformal higher spin fields in $A d S_{4}$ space. Thus, as the generalized AdS geometry of the free $A d S_{4}$ higher spin fields is described by the $O S p(1 \mid 4)$ supergroup manifold (and, likely, the scalar superfield on $O S p(1 \mid n)$ for $n=8,16$ describes the AdS massless conformal higher spin fields in $D=6,10$ as well), our construction indicates that the $A d S$-M-algebra is given by $\operatorname{osp}(1 \mid 32)$, in agreement with [43, 33, 34] (see also [35, 38]).

To see how to relate the AdS preon definition with the preservation of a fraction of the supersymmetries, we have discussed in Sec. 6 the superparticle model on the $O S p(1 \mid n)$ supergroup manifold [25, 27]. The ground state of this model preserves 31 supersymmetries associated with the 31 -parametric $\kappa$-symmetry of its action. Therefore, it is a BPS preon and the $O S p$ superparticle can be called an AdS preonic 0-brane. However, the action of this preserved part of the AdS supersymmetry on this BPS preonic state is $X$ (and $\theta$-) dependent, as it is the AdS supersymmetry acting on $O S p(1 \mid n)$ supermanifold. Thus, it is hard to see this preserved supersymemtry in the abstract (bra-ket) quantum mechanical language used to define the AdS preon (although there is no problem to describe it by a superwavefunction in the generalized coordinate representation). This explains why the preonic representation of the osp supersymmetry generators (20) cannot be obviously translated in terms of preserved supersymmetries and leads instead to a non-commutative deformation of the M-algebraic definition of the BPS preon, singling out the supersymmetry broken by the AdS preon. The appearance of a deformation is 
again not surprising if we recall that the Moyal brackets were introduced in higher spin theory [24] to describe the free $D=4$ higher spin theories in $A d S_{4}$ space.

The notion of the AdS preon introduced here suggests that the search for a dynamical mechanism to obtain the $k / 32$-BPS states from the BPS preons may be related to the problem of constructing a consistent interaction theory of a tower of massless conformal higher spin fields. Interacting, massless conformal higher spin theories were constructed in [23]. However, in our preonic context, we need a formulation of such an interacting theories in tensorial superspaces (see [7, 47, 48, 49, 4] for the free case). This is still unknown, although progress in this direction has been made by introducing higher spin gauge potentials in generalized AdS superspace [28].

A natural development of the present work would be to look for composites of AdS preons, in particular of $16 \mathrm{AdS}$ preons, corresponding to $1 / 2$-BPS states. From this point of view, it would be interesting to see whether one can give a non-commutative counterpart of e.g. the supermembrane BPS state and, if so, whether it would be related with the matrix model of a non-commutative membrane which is used to describe coincident M2branes (see [55] and refs. therein).

Acknowledgments. The authors thank Dima Sorokin for useful discussions. This work has been partially supported by research grants from the Ministerio de Educación y Ciencia (FIS2005-02761) and EU FEDER funds, the Generalitat Valenciana, the Ukrainian State Fund for Fundamental Research (N383), the INTAS (2006-7928) and by the EU MRTN-CT-2004-005104 'Forces Universe' network.

\section{References}

[1] I.A. Bandos, J.A. de Azcárraga, J.M. Izquierdo and J. Lukierski, BPS states in M-theory and twistorial constituents, Phys. Rev. Lett. 86, 4451-4454 (2001) arXiv:hep-th/0101113.

[2] I.A. Bandos, BPS preons and tensionless super-p-branes in generalized superspace, Phys. Lett. B558, 197-204 (2003) arXiv:hep-th/0208110.

[3] I.A. Bandos, J.A. de Azcárraga, M. Picón and O. Varela, D= 11 superstring model with 30 kappa-symmetries and 30/32 BPS states in an extended superspace, Phys. Rev. D69, 085007 (2004) arXiv:hep-th/0307106.

[4] I. Bandos, X. Bekaert, J. A. de Azcárraga, D. Sorokin and M. Tsulaia, JHEP 0505, 031 (2005) arXiv:hep-th/0501113.

[5] M.A. Vasiliev, Consistent equations for interacting massless fields of all spins in the first order in curvatures, Ann. Phys. (NY) 190, 59 (1989)

[6] D. Sorokin, Introduction to the classical theory of higher spins, AIP Conf. Proc. 767, 172 (2005) arXiv:hep-th/0405069.

[7] I. Bandos, J. Lukierski and D. Sorokin, Superparticle models with tensorial central charges, Phys. Rev. D61, 045002 (2000) arXiv:hep-th/9904109.

\footnotetext{
${ }^{9}$ For very recent progress in the description of supersymmetric non-commutative M2-branes see 53 . and [54].
} 
[8] I. Bandos and J. Lukierski, Tensorial central charges and new superparticle models with fundamental spinor coordinates, Mod. Phys. Lett. A14, 1257-1272 (1999) arXiv:hep-th/9811022.

[9] I. A. Bandos, J. A. de Azcárraga, J. M. Izquierdo, M. Picón and O. Varela, On BPS preons, generalized holonomies and D = 11 supergravities, Phys. Rev. D69, 105010 (2004) arXiv:hep-th/0312266.

[10] E. Cremmer, B. Julia and J. Scherk, Supergravity theory in eleven dimensions, Phys. Lett. B76, 409-412 (1978).

[11] P.K. Townsend, p-brane democracy, in Particles, strings and cosmology, J. Bagger et al. eds., World Sci. 1996, pp. 271-285 arXiv:hep-th/9507048; M-theory from its superalgebra, in Strings, branes and dualities, NATO ASI Ser. C, Math. and Phys. Sci., 520, 141-177 (1999) arXiv:hep-th/9712004, and refs. therein.

[12] I. Bandos and J. Lukierski, New superparticle models outside the HLS supersymmetry scheme, XII Max Born conf., Lect. Notes Phys. 539, 195 (2000) arXiv:hep-th/9812074.

[13] J. P. Gauntlett and C. M. Hull, BPS states with extra supersymmetry, JHEP 0001, 004 (2000) arXiv:hep-th/9909098.

[14] M. J. Duff, M-theory on manifolds of G(2) holonomy: The first twenty years, arXiv:hep-th/0201062.

[15] M. J. Duff and J. T. Liu, Hidden spacetime symmetries and generalized holonomy in Mtheory, Nucl. Phys. B674, 217 (2003) arXiv:hep-th/0303140;

M. J. Duff, 'The status of local supersymmetry, 2003 Erice lectures, arXiv:hep-th/0403160.

[16] C. Hull, Holonomy and Symmetry in M-theory, arXiv:hep-th/0305039.

[17] U. Gran, J. Gutowski, G. Papadopoulos and D. Roest, $N=31$ is not IIB, JHEP 0702, 044 (2007) arXiv:hep-th/0606049].

[18] I. A. Bandos, J. A. de Azcárraga and O. Varela, On the absence of BPS preonic solutions in IIA and IIB supergravities, JHEP 0609, 009 (2006) arXiv:hep-th/0607060.

[19] U. Gran, J. Gutowski, G. Papadopoulos and D. Roest, $N=31, D=11$, JHEP 0702, 043 (2007) arXiv:hep-th/0610331.

[20] J. Figueroa-O'Farrill and S. Gadhia, M-theory preons cannot arise by quotients, JHEP 0706, 043 (2007) arXiv:hep-th/0702055.

[21] U. Gran, J. Gutowski, G. Papadopoulos and D. Roest, IIB solutions with $N>28$ Killing spinors are maximally supersymmetric, arXiv:0710.1829 [arXiv:hep-th].

[22] I. A. Bandos and J. A. de Azcárraga, BPS preons in M-theory and supergravity, in Procs. of the 2nd RTN Network Workshop: Constituents, Fundamental Forces and Symmetries of the Universe, Naples, Italy, 9-13 Oct 2006, Fortsch. Phys. 55, 692-698 (2007) arXiv:hep-th/0702099.

[23] M. A. Vasiliev, Consistent equations for interacting massless fields of all spins in the first order in curvatures, Ann. Phys. (NY) 190, 59 (1989); Higher spin gauge theories in various dimensions, Fortsch. Phys. 52 (2004) 702 arXiv:hep-th/0401177];

X. Bekaert, S. Cnockaert, C. Iazeolla and M. A. Vasiliev, Nonlinear higher spin theories in various dimensions, arXiv:hep-th/0503128. 
[24] M. A. Vasiliev, Higher spin gauge theories: star-product and AdS space, in The many faces of the superworld, Yu. Golfand's Memorial Volume, M. Shifman ed., pp. 533-610, World Scientific, 2000 arXiv:hep-th/9910096 and refs. therein.

[25] I. A. Bandos, J. Lukierski, C. Preitschopf and D. P. Sorokin, OSp supergroup manifolds, superparticles and supertwistors, Phys. Rev. D61 (2000) 065009 arXiv:hep-th/9907113.

[26] V.E. Didenko and M.A. Vasiliev, Free field dynamics in the generalized AdS (super)space, J. Math. Phys. 45, 197 (2004) arXiv:hep-th/0301054.

[27] M. Plyushchay, D. Sorokin and M. Tsulaia, Higher spins from tensorial charges and $O S p(N \mid 2 n)$ symmetry, JHEP 0304, 013 (2003) arXiv:hep-th/0301067]; GL flatness of $O S p(1 \mid 2 n)$ and higher spin field theory from dynamics in tensorial spaces, in Supersymmetries and Quantum Symmetries (SQS 03), Dubna, Russia, 24-29 Jul 2003, arXiv:hep-th/0310297.

[28] M. A. Vasiliev, On Conformal, $S L(4, R)$ and $S p(8, R)$ Symmetries of $4 d$ Massless Fields, arXiv:0707.1085 [hep-th]

[29] J. Figueroa-O'Farrill and G. Papadopoulos, Maximally supersymmetric solutions of tenand eleven-dimensional supergravities, JHEP 0303, 048 (2003) arXiv:hep-th/0211089.

[30] O. Baerwald and P. C. West, Brane rotating symmetries and the fivebrane equations of motion, Phys. Lett. B476, 157-164 (2000) [arXiv:hep-th/9912226];

J. P. Gauntlett, G. W. Gibbons, C. M. Hull and P. K. Townsend, BPS states of D $=4 \mathrm{~N}$ = 1 supersymmetry, Commun. Math. Phys. 216, 431-439 (2001) arXiv:hep-th/0001024.

[31] J. A. de Azcárraga, J. M. Izquierdo, M. Picón and O. Varela, Generating Lie and gauge free differential (super)algebras by expanding Maurer-Cartan forms and Chern-Simons supergravity, Nucl. Phys. B662, 185 (2003) arXiv:hep-th/0212347]; Expansions of algebras and superalgebras and some applications, in Proc. of Deformations and Contractions in Mathematics and Physics, Oberwolfach Math. Center, Jan. 2006, Int. J. Theor. Phys. 46, 2938-2752 (2007) arXiv:hep-th/0703017.

[32] C. Chryssomalakos, J.A. de Azcárraga, J.M. Izquierdo and J.C. Pérez Bueno, The geometry of branes and extended superspaces, Nucl. Phys. B567, 293-330 (2000) arXiv:hep-th/9904137;

J.A. de Azcárraga and J.M. Izquierdo, Superalgebra cohomology, the geometry of extended superspaces and superbranes, in New developments in fundamental interactions theories, 37th Karpacz Winter School, AIP Conf. Proc. 589, 3-17 (2001) arXiv:hep-th/0105125.

[33] M. Gunaydin and D. Minic, Singletons, doubletons and M-theory, Nucl. Phys. B 523, 145 (1998) arXiv:hep-th/9802047;

M. Gunaydin, Unitary supermultiplets of $\operatorname{OSp}(1 / 32, R)$ and M-theory, Nucl. Phys. B 528, 432 (1998) arXiv:hep-th/9803138;

[34] S. Ferrara and M. Porrati, AdS(5) superalgebras with brane charges, Phys. Lett. B 458, 43 (1999) arXiv:hep-th/9903241;

[35] E. Bergshoeff and A. Van Proeyen, The many faces of $O S p(1 \mid 32)$, Class. Quant. Grav. 17, 3277 (2000) arXiv:hep-th/0003261; The unifying superalgebra OSp(1|32), in Moscow 2000, Quantization, gauge theory, and strings, vol. 1, pp. 48-59 arXiv:hep-th/0010194. 
[36] I. Bars, $2 T$ physics formulation of superconformal dynamics relating to twistors and supertwistors, Phys. Lett. B483, 248-256 (2000) arXiv:hep-th/0004090];

I. Bars, C. Deliduman and D. Minic, Lifting M-theory to two-time physics, Phys. Lett. B457, 275-284 (1999) arXiv:hep-th/9904063.

[37] P. Horava, M-theory as a holographic field theory, Phys. Rev. D 59, 046004 (1999) arXiv:hep-th/9712130.

[38] K. Kamimura and M. Sakaguchi, osp (1|32) and extensions of super-Ad $S_{5} \times S^{5}$ algebra, Nucl. Phys. B 662, 491 (2003) arXiv:hep-th/0301083;

[39] P. Meessen, K. Peeters and M. Zamaklar, On non-perturbative extensions of anti-de-Sitter algebras, arXiv:hep-th/0302198;

K. Peeters and M. Zamaklar, Anti-de-Sitter vacua require fermionic brane charges, Phys. Rev. D 69, 066009 (2004) arXiv:hep-th/0311110.

[40] R. D'Auria and P. Fré, Geometric supergravity In D = 11 and Its hidden supergroup, Nucl. Phys. B201, 101-140 (1982) [Erratum: ibid. B206, 496 (1982)].

[41] I. A. Bandos, J. A. de Azcárraga, J. M. Izquierdo, M. Picón and O. Varela, On the underlying gauge group structure of $D=11$ supergravity, Phys. Lett. B596, 145 (2004) arXiv:hep-th/0406020;

I. A. Bandos, J. A. de Azcárraga, M. Picón and O. Varela, On the formulation of $D=$ 11 supergravity and the composite nature of its three-form field, Annals Phys. 317, 238 (2005) arXiv:hep-th/0409100.

[42] J. W. van Holten and A. Van Proeyen, $N=1$ Supersymmetry algebras in $D=2, D=3, D=4$ mod-8, J. Phys. A 15, 3763 (1982).

[43] P. K. Townsend, M(embrane) theory on $T^{9}$, Nucl. Phys. Proc. Suppl. 68, 11-16 (1998) arXiv:hep-th/9708034.

[44] J.A. de Azcárraga, J.P. Gauntlett, J.M. Izquierdo and P.K. Townsend, Topological extensions of the supersymmetry algebra for extended objects, Phys. Rev. Lett. 63, 213 (1989).

[45] C. M. Hull, Gravitational duality, branes and charges, Nucl. Phys. B509, 216 (1998) arXiv:hep-th/9705162.

[46] D. P. Sorokin and P. K. Townsend, M-theory superalgebra from the M-5-brane, Phys. Lett. B412, 265 (1997) arXiv:hep-th/9708003.

[47] M.A. Vasiliev, Conformal higher spin symmetries of $4 d$ massless supermultiplets and osp $(L, 2 M)$ invariant equations in generalized (super)space, Phys. Rev. D66, 066006 (2002) arXiv:hep-th/0106149.

[48] M.A. Vasiliev, Relativity, causality, locality, quantization and duality in the $S p(2 M)$ invariant generalized space-time, in Multiple facets of quantization and supersymmetry, Marinov's memorial volume, M.Olshanetsky and A.Vainshtein eds., p. 826 arXiv:hep-th/0111119.

[49] I. Bandos, P. Pasti, D. Sorokin and M. Tonin, Superfield theories in tensorial superspaces and the dynamics of higher spin fields, JHEP 0411, 023 (2004) arXiv:hep-th/0407180.

[50] D. P. Sorokin, Supersymmetric particles, classical dynamics and its quantization, Preprint ITP-87-159, Kiev, 1988 [unpublished]; for a discussion see [7, 49]. 
[51] J. A. de Azcárraga, J. M. Izquierdo and P. K. Townsend, A Kaluza-Klein origin for the superstring tension, Phys. Rev. D 45, 3321 (1992);

P. K. Townsend, World sheet electromagnetism and the superstring tension, Phys. Lett. B277, 285 (1992);

E. Bergshoeff, L. A. J. London and P. K. Townsend, Space-time scale invariance and the super-p-brane, Class. Quant. Grav. 9, 2545 (1992) arXiv:hep-th/9206026];

I. A. Bandos, D. P. Sorokin, M. Tonin and D. V. Volkov, Doubly supersymmetric null strings and string tension generation, Phys. Lett. B 319, 445 (1993) arXiv:hep-th/9307039;

E. Bergshoeff, D. P. Sorokin and P. K. Townsend, The M5-brane Hamiltonian, Nucl. Phys. B 533, 303 (1998) arXiv:hep-th/9805065];

J. A. de Azcárraga, J. M. Izquierdo and C. Miquel-Espanya, Spacetime scale-invariant super-p-brane actions on enlarged superspaces and the geometry of kappa-symmetry, Nucl. Phys. B706, 181 (2005) arXiv:hep-th/0407238.

[52] E. S. Fradkin and M. A. Vasiliev, Cubic Interaction in Extended Theories of Massless Higher Spin Fields, Nucl. Phys. B291, 141 (1987); E. S. Fradkin and M. A. Vasiliev, On the Gravitational Interaction of Massless Higher Spin Fields, Phys. Lett. B189, 89 (1987).

[53] J. Bagger and N. Lambert, Modeling multiple M2's, Phys. Rev. D75, 045020 (2007) arXiv:hep-th/0611108; Gauge Symmetry and Supersymmetry of Multiple M2-Branes, arXiv:0711.0955 [hep-th]; Comments On Multiple M2-branes, arXiv:0712.3738 [hep-th].

[54] A. Gustavsson, Algebraic structures on parallel M2-branes, arXiv:0709.1260 [hep-th].

[55] D. S. Berman, M-theory branes and their interactions, arXiv:0710.1707 [hep-th]. 